\title{
La neuro-génétique
}

La «neuro-génétique», c'est-à-dire la génétique du système nerveux, a bénéficié, comme les autres disciplines biologiques et médicales, de l'irruption des méthodes de recombinants d'ADN. L'expression de transcrits d'oncogènes et d'autres gènes du développement a été étudiée au cours de l'organisation du système nerveux central. Les gènes responsables de certaines maladies neurologiques ont été localisés, voire clonés et identifiés. De premiers résultats de correction par transfert de gène de modèles animaux de ces maladies ont été publiés. Le système nerveux est cependant si complexe, dans son organisation et sa fonction, qu'il serait néanmoins naïf de croire que l'identification de tous les gènes impliqués dans son développement (des milliers ou des dizaines de milliers) suffira à éclairer l'ensemble de ces processus. Les problèmes de communication et de signalisation intercellulaire, ainsi que l'influence du contexte psychologique et du milieu humain, restent ici des données essentielles non contenues dans l'enchaînement des bases de l'ADN.

\section{Jacques-Michel Robert}

Professeur de génétique à la faculté de médecine Lyon-Sud. Chef du service de génétique de l'Hôtel-Dieu de Lyon

\section{RÉFÉRENCES}

1. Robert JM. Développement humain. Encyclopaedia Universalia 1985 ; 6: 46-58.

2. Pavans de Ceccaty M. La vie de la cellule à l'homme. Paris : Le Seuil, 1962.

3. Duhamel B, HaegelP, Pages R. Morphogenèse pathologique. Des monstruosités aux malformations. Paris: Masson, 1966.

\section{ADRESSE}

J.-M. Robert: Hôtel-Dieu, service de génétique, place de l'Hôpital, 69002 Lyon, France. $m / s n^{\circ} 2$ vol. 4, mars 88 l est possible, sans trop d'artifice, d'identifier neurogénétique et développement du système nerveux pris dans son sens le plus large, normal ou pathologique, de l'embryogenèse à la mort de l'individu [1]. La neurulation achevée, les cellules du neuro-épithélium se multiplient et migrent en direction de leurs cibles respectives. Dendrites et axones se rapprochent et réalisent la synaptogenèse. La myéline apparaît et la myélinisation sera en perpétuel renouvellement. Les neurones en place reliés à d'innombrables congénères vont synthétiser neurotransmetteurs et neuropeptides. Ils fonctionnent, et de ce fonctionnement dépendront motricité, sensibilité mais aussi mémoire, apprentissage, humeurs, comportements. Puis les rangs de la population neuronale s'éclairciront avec l'âge, et parfois «avant l'âge». La maintenance et la sénescence du système nerveux central (SNC) ont partie liée. La recherche en neuro-génétique a marqué des points, ces dernières années, dans tous ces domaines.

\section{Neurulation}

L'insecte serait-il un homme qui a mal tourné ou bien l'homme un insecte qui a bien tourné? Audelà de cette boutade de Max de Ceccatty - exprimée dans l'un de ses premiers ouvrages qui n'a pas une ride [2] - surgit ce rappel que le règne animal propose deux grands modèles de SNC (système nerveux central): ou bien l'on porte celui-ci sur le ventre et l'on est un hyponeurien, avec pour destin classificatoire de rester à jamais un être sans vertèbre, ou bien on le porte sur le dos et l'on sera épineurien. Une noto- 


\section{RÉFÉRENCES}

4. Wilson DB, Darlene PW. Pathogenesis of neural dysraphism in the mouse mutan vacuolated lens (vl). J Neuropathol Exp Neurol 1986 ; 45 : 43-55.

5. Robert E, Guibaud P. Maternal valproïc acid and congenital neural tube defects. Lancet 1982 ; ii : 937.

6. Wild J, Read AP, Sheppard S, et al. Recurrent neural tube defects, risk factors and vitamins. Arch Dis Child 1986; 61 : 440-4.

7. Sadler TW, Greenberg D., Coughlin P. Actin distribution patterns in mouse neural tube during neurulation. Science $1982 ; 215$ : 172-4.

8. Wilkinson DG, Balles JA, McMahon AP Expression of the proto-oncogene Intl is restricted to specific neural cells in the developing mouse embryo. Cell 1987 ; 50 : 79-88.

9. Guerrero I. Proto-oncogens in pattern formation. Trends Genet 1987; $3: 269-71$.

10. Cowie VA. Microcephaly : a review of genetic implications in its causation. J Ment Defic Res 1987 ; 31 : 229-33.

11. Levi-Montalcini $R$. The nerve growth factor. 35 years later. Science 1987 ; 237 : 1154 62

12. Taniuchi M, Schweitzer JB, Johnson EM Jr. Nerve growth factor receptor molecules in rat brain. Proc Natl Acad Sci USA 1986 ; 83 1950.

13. Morrison RS, Kornblum HI, Leslie FM, Bradshaw RA. Trophic stimulation of cultured neurons from neonatal rat brain by EGF Science 1987 ; 238 : 72-5.

14. Le Douarin N. The neural crest. Developmental and cell biology, vol. 12. Cambridge : University Press, 1983 .

15. Takeichi M. Cadherins: a molecular family essential for selective cell-cell adhesion and animal morphogenesis. Trends Genet $1987 ; 3: 213-7$

16. Morris-Kay G, Seong-Seng Tan. Mapping cranial neural crest cell migration pathways in mammalian embryos. Trends Genet $1987 ; 3: 257-261$

17. Seizinger BR, Rouleau GA, Ozelius LJ, et al. Genetic linkage of von Recklinghausen neurofibromatosis to the Nerve Growth factor Receptor gene. Cell 1987 ; 49 : 589-94.

18. Seizinger BR, et al. Common paihogenic mechanism for three tumor types in bilateral acoustic neurofibromatosis. Science 1987 ; 236 : $317-9$.

19. Hinrinchs SH, Nerenberg M, Kay Reynolds R, Khoury G, Jay G. A transgenic mouse model for human neurofibromatosis.

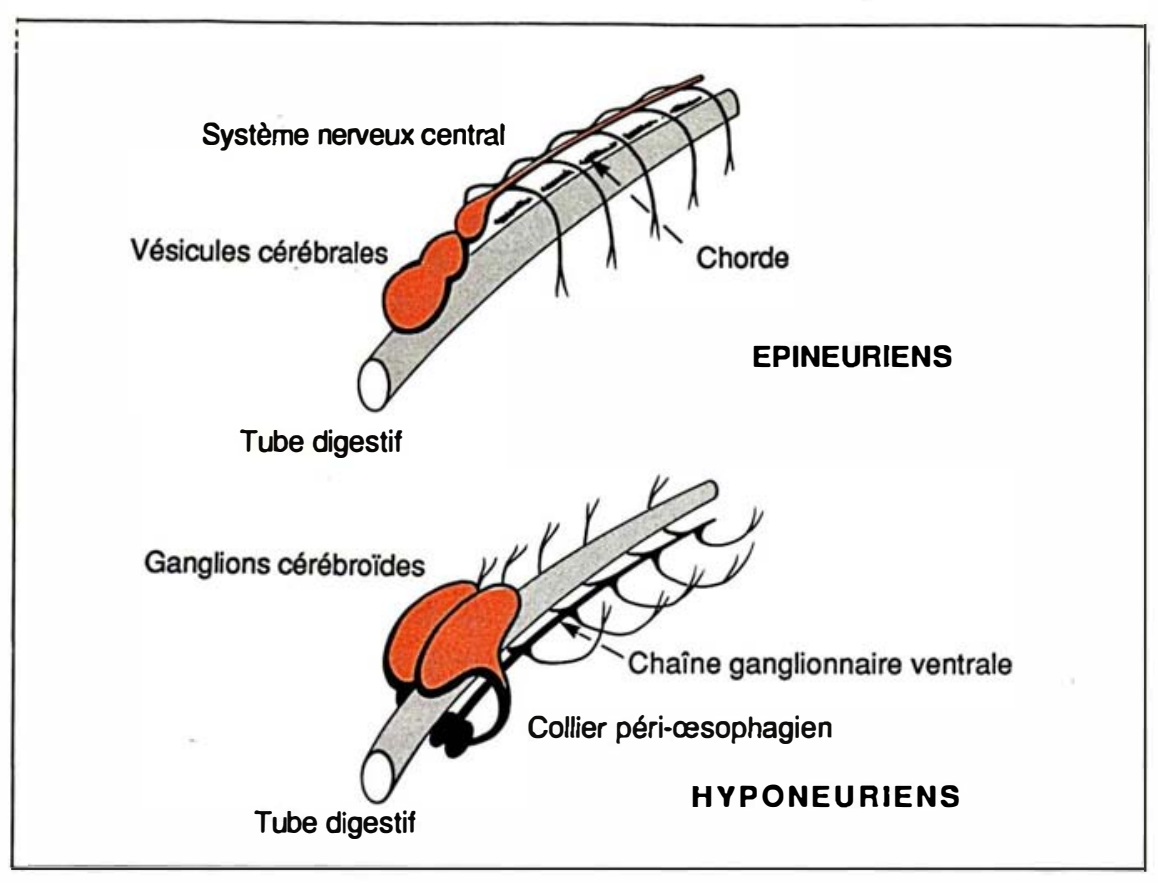

Figure 1. Le système nerveux central chez les épineuriens et chez les hyponeuriens (d'après [2]).

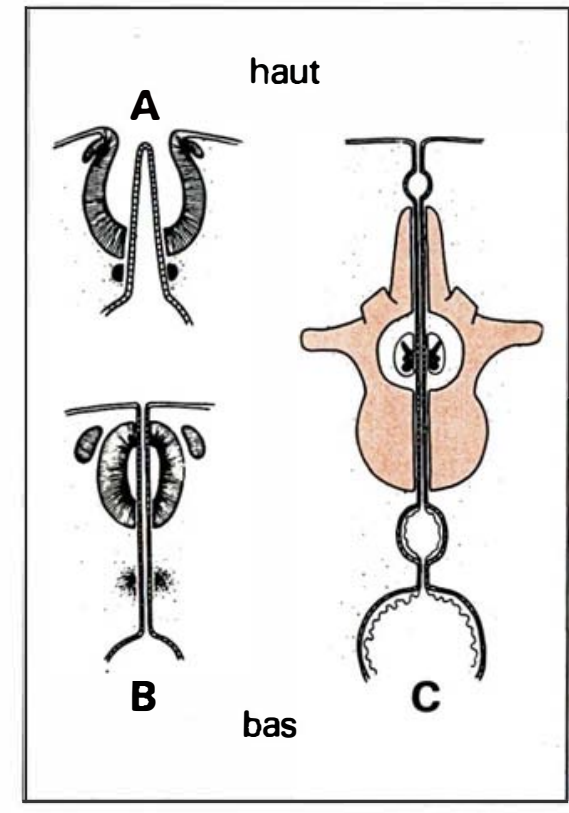

Figure 2. Coupe sagittale schématique du processus de la notochordodysraphie. (A). Au stade de la gouttière neurtale. (B). Au stade du tube neural. (C). Au stade définitif (fistule entérique dorsale avec diastématomyélie). chorde* induira la formation d'une plaque, puis d'une gouttière, d'un tube enfin, germe luimême de l'ampoule cérébrale. Un étui osseux d'origine conjonctive (vertèbres et crâne) naîtra pour protéger ces merveilles. Strate après strate, la poussée vers l'avant du cerveau des épineuriens n'a rencontré nulle entrave alors que, lors d'une semblable poussée, le système nerveux central de l'hyponeurien s'est heurté au redoutable développement des pièces olfactives et buccales et n'a pu caser ses «ganglions cérébroïdes », fragilement reliés à lui par un collier péri-œsophagien, qu'à l'intérieur d'une tête minuscule revêtue de chitine (figure 1). L'homme, lui, est un épineurien, chordé, neurulé, vertébré, cérébré, corticalisé, frontalisé.

On doit à B. Duhamel[3] le concept de noto-chordodysraphie. Cette première catastrophe de la neuro-embryogenèse est datée,

\footnotetext{
* Du grec notos, le dos, et chordè, boyau.
} 


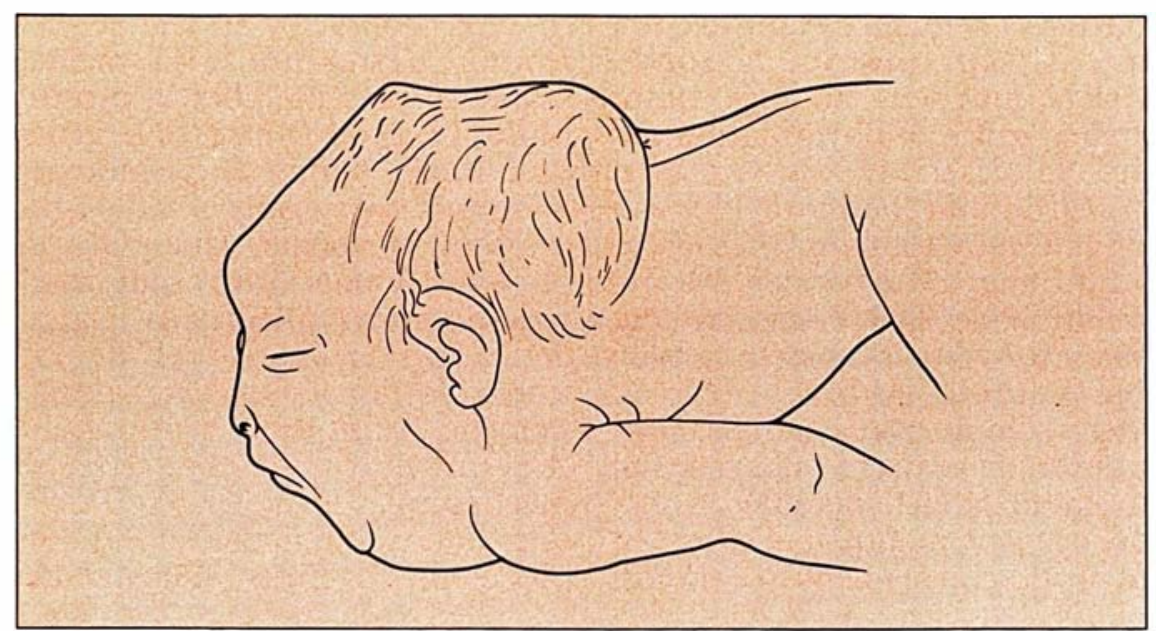

Figure 3. Iniencéphalie. Inion = protubérance occipitale externe. (Schéma emprunté à B. Duhamel [3]).

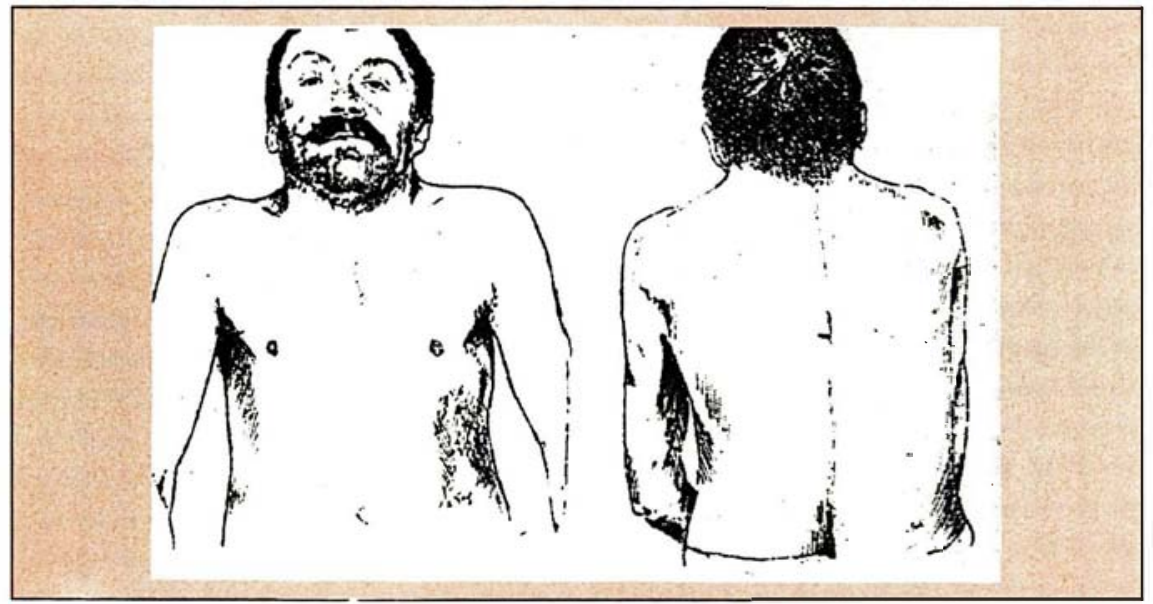

Figure 4. Syndrome des "hommes sans cou", décrit par Klippel-Feil en 1912. (Schéma original tiré de [47]).

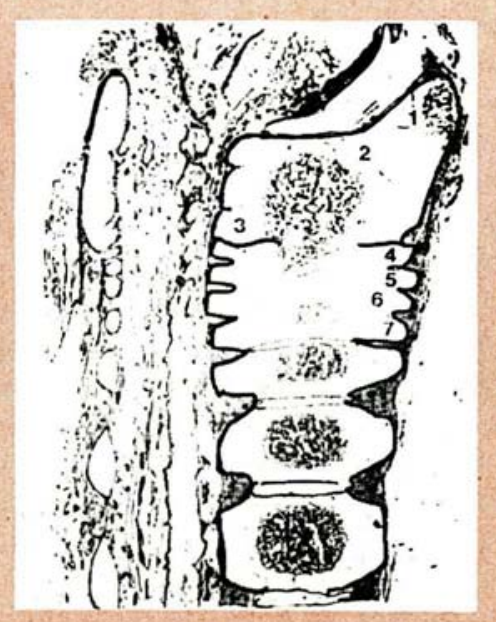

Figure 5. Fœtus $(30 \mathrm{~cm})$ d'une baleine. Absence de disques entre les vertèbres C3 à C6 (tiré de [48]).

$m / s n^{\circ} 3$ rol. + , mars 88 lorsqu'elle survient, de la troisième semaine du développement. Les anomalies de l'induction chordale expliquent la diplomélie (double moelle), la diastématomyélie (moelle unique partagée en deux par un éperon cartilagineux vestigial), ou l'exceptionnelle persistance d'un canal neurentérique avec issue de méconium dans la région dorsale (figure 2). L'iniencéphalie (figure 3) est la forme extrême de l'anomalie décrite en 1912 par KlippelFeil sous le nom d' " hommes sans cou» (figure 4): les blocs cervicaux (hérités habituellement en dominance autosomique) résultent du défaut de pénétration entre les vertèbres des structures ultimes de la chorde dorsale (nucleus pulposus du disque intervertébral). Cette construction est physiologique chez certains cétacés (figure 5).

A la fin de la quatrième semaine, la neurulation doit être achevée. Dans le cas contraire, il y a nonfermeture du tube neural (NFTN), dite encore neuro-dysraphie. L'absence de développement de l'ampoule antérieure conduit à l'anencéphalie toujours létale. A l'autre extrémité du tube neural, la non-fermeture crée le spina bifida lombaire dont il existe différents degrés de gravité (figure 6, page 160). L'amphioxus (en grec signifie: pointu aux deux bouts) est un prochordé «fossile vivant» qui présente à l'état naturel anencéphalie et spina bifida. Un mutant «naturel» dit vl existe chez la souris. Le phénotype de l'homozygote pour le gène muté peut présenter tous les degrés possibles de neurodysraphie [4]. Surtout, il existe dans l'espèce murine, sur le chromosome 17, en étroite relation avec la région $\mathrm{H} 2$, une série de loci dite système $T / t$ (tail) dont les diverses mutations dysraphiantes sont bien connues. La présence d'un système génétique comparable dans notre espèce, au sein des loci de la famille MHC est possible mais non établie de façon absolue. Quoi qu'il en soit, il existe en ce domaine un 
«terrain prédisposant»: les NFTN sont nettement plus fréquentes en Irlande, Écosse, Pays de Galles que partout ailleurs dans le monde (ethnie celte?). Par ailleurs, il a été établi que l'usage de l'acide valproïque dans le traitement de l'épilepsie chez les femmes enceintes augmente fortement le risque de neuro-dysraphie chez le fœetus [5]. A l'inverse, l'utilisation pré-conceptionnelle et périconceptionnelle de traitements polyvitaminés, à base d'acide folique notamment, réduirait ce même risque [6].

Deux types de recherche à cinq ans d'intervalle marquent l'intérêt porté par les expérimentateurs au phénomène de la neurulation. En 1982 Sadler et al. ont utilisé des anticorps anti-actine et les techniques d'immunofluorescence indirecte pour suivre, au cours de la neurulation chez l'embryon de la souris, la localisation cellulaire des microfilaments selon le temps de l'embryogenèse (stade 2 somites à stade 5 somites). Sur les coupes transversales successives les lèvres du neuro-épithélium sont en quasi-contact d'abord parallèles, sinon d'aspect légèrement éversé: l'actine des microfilaments est alors électivement localisée à la base des neurones. A l'instant de la neurulation, l'actine se concentre à l'apex des cellules nerveuses et ceci en deux points : tout en haut du tube qui bientôt se détachera de l'épiblaste; tout en profondeur de celui-ci, au niveau du sillon primitif en cet autre point où les deux lèvres devront connaître un contact définitif. Sur la préparation, l'ensemble tend à perdre sa forme biconvexe obligée : la tubulation est obtenue [7].

Une seconde série d'expériences, plus récente, appelle l'attention sur l'étroite parenté sinon l'identité entre certains proto-oncogènes et certaines séquences génétiques indispensables à l'embryogenèse normale d'un vertébré. Wilkinson et al. ont focalisé leur attention sur le proto-oncogène Int.1 [8] antérieurement connu pour sa présence au sein de cer- souris : sa séquence étant déchiffrée, ils ont construit la sonde d'ARN anti-sens adaptée pour repérer grâce à l'hybridation in situ la distribution spatiale d'Int.1 du jour 9 au jour 14,5. Le résultat est étonnant (figure 7): durant toute cette période, l'accumulation de l'ARN transcrit du gène Int.1 reste localisée à la plaque neurale et à ses dérivés directs. On ne trouve jamais d'expression de ce proto-oncogène au niveau de la crête neurale.

On ne peut manquer d'effectuer un rapprochement entre ce travail et ceux consacrés à l'étude de D.int 1, c'est-à-dire à la même séquence présente chez la drosophile. Le gène $D$-int 1 et le gène qui, muté, donne le phénotype wingless (wg) sont, semble-t-il, un seul et même gène. Or ce phénotype extériorise toute une série de graves altérations létales de la polarisation et de la «dorsalisation» des larves de la mouche. Avant toute neurulation chez un vertébré (et chez certains invertébrés), des molécules identiques interviendraient déjà lors des processus les plus anciens du

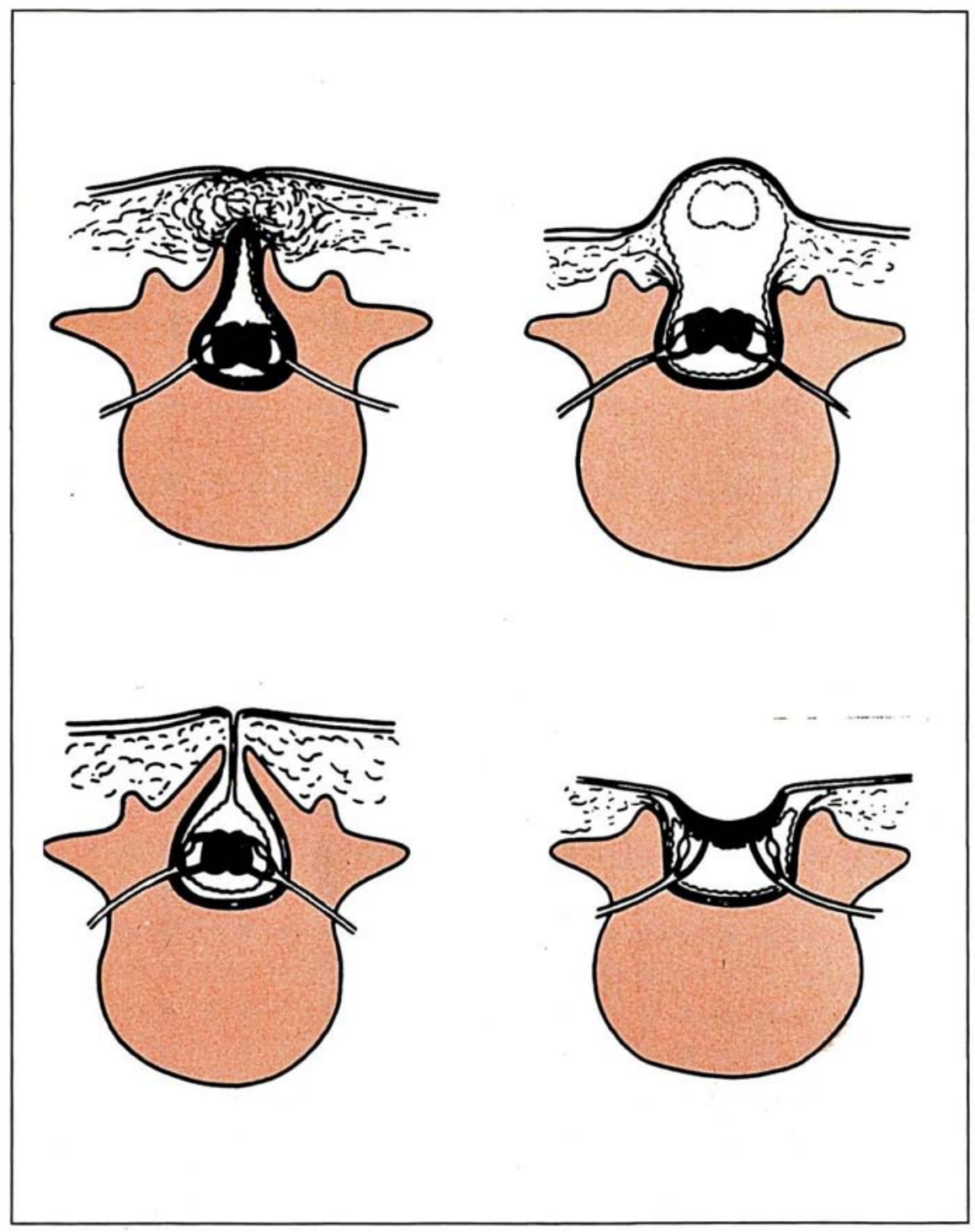

Figure 6. Les différents degrés de sévérité d'un spina bifida lombaire. A gaucho, en haut: spina bifida occulta; en bas: sinus dermique. A droite, en haut: méningocèle et/ou myélocèle; en bas: spina bifida aperta (ulcéré, sans épidermisation). 


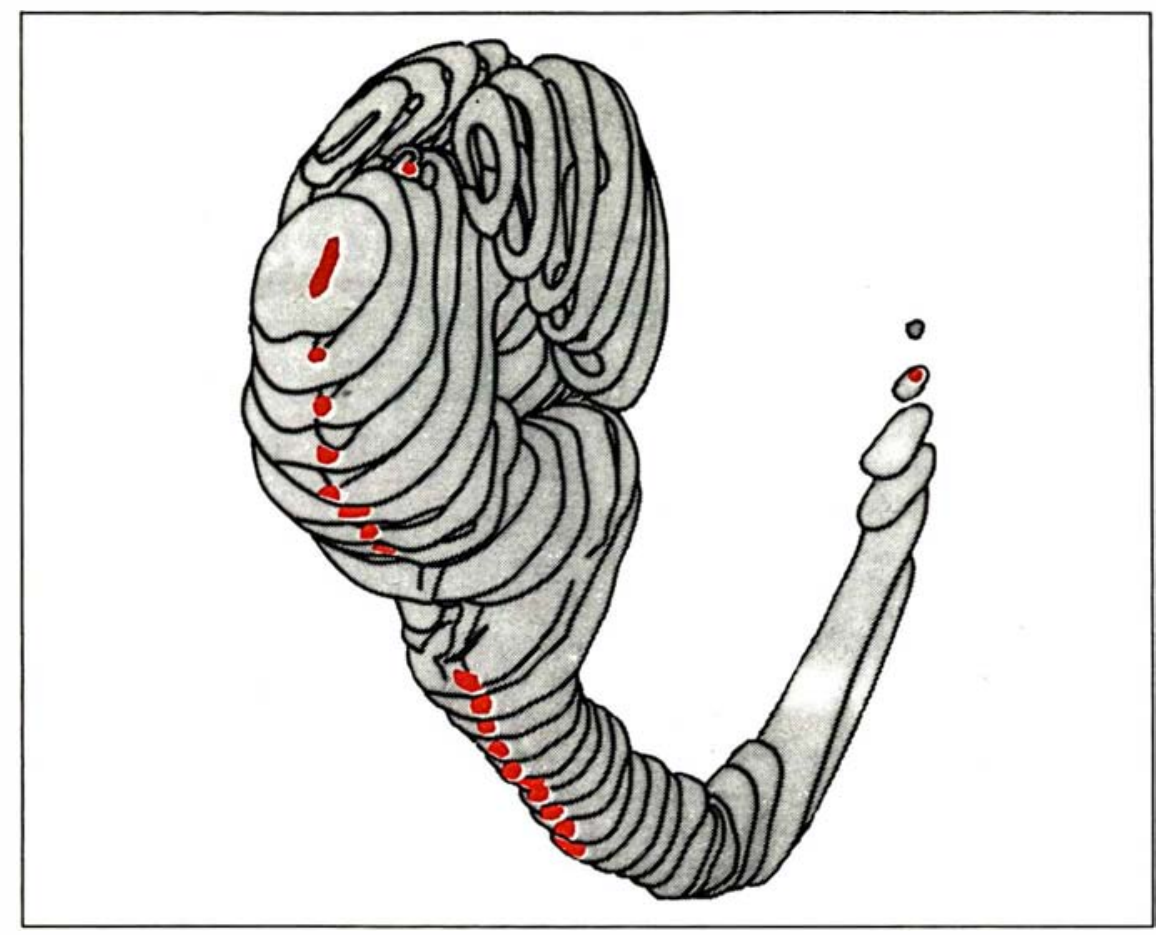

Figure 7. Expression int.1 au niveau du tube neural de la souris (Schéma inspiré de la couverture de la revue Cell, juillet 1987).

neuro-développement, peut-être en tant que facteurs de croissance, récepteurs de facteurs de croissance, G-like protéines impliquées dans la transduction d'un signal [9].

\section{Multiplication}

Selon certaines estimations, difficiles à prouver, le neuro-épithélium du tube neural assurerait 250000 mitoses à la minute entre le deuxième et la fin du quatrième mois fotal. Malgré les progrès récents accomplis dans les recherches à propos des facteurs de croissance cellulaire, la neurogénétique rencontre ici plus d'interrogations que de réponses. Quelle est l'expression du gène humain, transmis en récessivité autosomique, qui provoque la Microcephalia vera ? Ces « cerveaux miniatures » sont classiquement bien formés. Des études histopathologiques plus précises révèlent bien souvent hétérotopies cellulaires, désordres architectoniques du cortex, anomalies des sillons et circonvolutions [10]. Les $\mathrm{m} / \mathrm{s} n^{\circ} 3$ vol. 4 , mars 88 atténuée de la précédente. Il existe deux ventricules latéraux mais le troisième ventricule est très dilaté. L'anomalie est viable et parfois peu démonstrative, découverte à la suite de l'apparition de crises épileptiques et d'une hydrocéphalie. Il existe aussi des agénésies partielles du corps calleux dont la transmission est liée à l'X (McKusick $\left.\mathrm{n}^{\circ} 30410\right)^{* * *}$.

La source de ces désordres neuronaux d'origine mitotique aurait pu être espérée dans une défaillance des facteurs de croissance. Il semble qu'il n'en soit rien. Le NGF (nerve growth factor) est un facteur neuronotrophique et de différenciation [11]. Il n'est pas mitogène. S'il intervient au cours du développement, c'est dans la sphère des neurones sensoriels et sympathiques du système nerveux périphérique. Plus tard, comme le souligne Eugène Johnson [12]: «NGF est synthétisé dans le cerveau, le message est dans le cerveau où il existe des récepteurs qui l'attendent. Il est difficile d'imaginer que cela ne serve à rien ». Mais, en l'état actuel des recherches, il est difficile d'aller au-delà.

L'EGF (epidermal growth factor) aide à l'ouverture des paupières des nouveau-nés mammifères, à la fermeture de leur voûte palatine, à la poussée dentaire. Il semble, selon Morrison et al., qu'il contribue aussi à la survie des neurones du néocortex et qu'il stimule leur développement chez le rat nouveau-né [13].

\section{Migrations et synaptogenèse} l'échelle génique transmise en récessivité autosomique.

L'agénésie du corps calleux peut être entendue comme une forme

\footnotetext{
* Eil unique dans une orbite unique.

* Yeux à demi fusionnés dans une orbile unique.

*** In McKusick V.Mendelian inhevilance in man, $7^{e}$ ed. The John Hopkins University Press, 1986, 1741 pages.
}

- Migrations. Tout a été dit à propos du destin de la crête neurale chez les oiseaux dans le sillage des remarquables travaux de Nicole le Douarin et al. [14]. Le rôle fondamental de la fibronectine est apparu $\left(\mathrm{m} / \mathrm{s} n^{\circ} 6, v o l .3\right.$, p. 314, éditorial de L. Degas et A. Kahn, et p. 316, article de synthèse de J.-P. Thiéry et al.). Il 


\section{REEFÉRENCES}

20. Griffiths DFR, Williams GT, Wil liams ED. Multiple endocrine neoplasia associated with von Recklinghausen's disease. $\mathrm{Br}$ Med J 1983 ; 287 : 1341-3.

21. Fryer AE, Chalmers A, Connor JM, et al. Evidence that the gene for tuberous sclerosis in on chromosome 9. Lancet 1987 ; i : 659-61.

22. Hausser I, Anton-Lamprecht I. Electron microscopy as a mean for carrier detection and genetic counseling in families at risk of tuberous sclerosis. Hum Genet 1987 ; 76 : 73 80.

23. Rakic P. Cell migration and neuronal ectopias in the brain. Birth defects. Original article series, vol. $11, n^{\circ} 7$. The National Foundation. New York: Alan R. Liss Inc, 1975 : 95-129.

24. Samat HB. Disturbance of late neuronal migrations in the perinatal period. $A m J D$ is Child 1987; 141 : 969-80.

25. Dobyns WB. Developmental aspects of lissencephaly and the lissencephaly syndromes. Birth Defects. Original article series, vol. 23, $n^{\circ}$ l. March of Dimes : Birth Defects Foundation. New York: Alan R. Liss Inc, $1987: 225-41$

26. Schutgens RBH, Heymans HSA, Wanders RJA, et al. Peroxisomal disorders : a newly recognised group of genetic diseases. Eur J Pediatr 1986; 144 : 490-40

27. Sotelo C, Alvarado-Mallart RM. Embryonic and adult neurons interact to allow Purkinje cell replacement in mutant cerebellum. Nature 1987 ; 327 : 421-3.

28. Mendlewica J, Simon Ph, Sevy S, et al. Polymorphic DNA marker on X chromosome and manic depression. Lancet 1987 ; i : 1230 -

29. Egeland JA, Gerhard DS, Pauls DL. Bipolar affective disorders linked to DNA markers on chromosome 1l. Nature 1987 . 325 : 783-7.

30. Berod A, Faucon-Biguet N, Dumas S, Bloch B, Mallet J. Modulation of tyrosine hydroxylase gene expression in the central nervous system visualized by in situ hybridization. Proc Natl Acad Sci USA 1987; 84 : 1699-703.

31. Hodgkinsons, Sherrington R, Gurling $H$, et al. Molecular genetic evidence for heterogeneity in manic depression. Nature 1987 ; \$25 : 805-6.

32. Baumann N. Neurological mutations affecting myelinisation. Amsterdam : Elsevier North Holland Biomed Press, 1980.

33. Roach A, Takahasi N, Prautecheva D, Ruddle F, Hood L. Chromosomal mapping of mouse myelin basic protein gene and structure and transcription of the partially deleted gene in shiverer mutant mice. Cell 1985 ; 42 : 149-55. existe, lors de la migration des cellules de la crête neurale, durant la cinquième semaine de l'embryogenèse, une simultanéité entre la chute des N-CAM (facteur d'adhérence) et la synthèse de la fibronectine. M. Takeichi [15] de Kyoto étudie, en ce qui le concerne, les cadhérines, famille de molécules qui semblent jouer un rôle considérable dans l'adhésion cellulaire. Sur le plan de leur structure antigénique, les cadhérines seraient proches des CAM étudiés par le laboratoire de Gérald Edelman de l'Institut Rockefeller (Pour la Science 1984, $\left.n^{\circ} 80, p .92-104\right)$. Après un trajet en zig-zag lié aux bonnes ou aux mauvaises rencontres avec d'autres éléments du même « torrent », ou avec les barrières physiques que sont des structures tissulaires d'un tout autre type, les cellules de la crête (Tableau I) gagnent leur objectif définitif topologique et fonctionnel (homing) à la vitesse moyenne de 40 micromètres à l'heure. Le taux de fibro- nectine dans leur environnement s'effondre, alors que celui des molécules N-CAM remonte. S'ensuivent l'arrêt et l'agrégation des cellules, liés à une différenciation exprimée dans leur phénotype par un changement évident de leur aspect.

Les travaux les plus récents sont du domaine expérimental et bioclinique. G. Morris-Kay et SeongSeng Tan ont développé une étude chez les embryons de rat à partir de micro-injections de cellules de la crête marquées à la thymidine tritiée [16]. Cette publication confirme les conclusions tirées de l'étude de la chimère caille-poulet, à quelques nuances près : chez les mammifères, les cellules de la crête émigrent à partir des lèvres d'un sillon encore largement ouvertes et non pas une fois la neurulation achevée. Aucune cellule ne semble émigrer de la crête à destination du cerveau antérieur et le mouvement chronologique n'est pas rosto-caudal comme il l'est chez l'oiseau.
Tableau I

LES CELLULES DÉRIVÉES DE LA CRÊTE NEURALE

Cellules nerveuses

- Ganglions sensoriels de certains nerfs crâniens

- Ganglions spinaux

- Cellules ganglionnaires du système nerveux autonome

Cellules associées

- Cellules gliales

aux neurones

- Cellules de Schwann

Méninges

Cellules pigmentaires

Cellules endocrines ou para-endocrines

- Cellules adrénomédullaires et autres cellules paraganglionnaires adrénergiques

- Cellules à calcitonine

- Cellules type I du corps carotidien

Dérivés mésodermiques

- Squelette de la face

- Squelette viscéral

- Parois des troncs aortiques

- Tissu conjonctif du thymus et des glandes parathyroïdes

- Derme du cou et de la face 


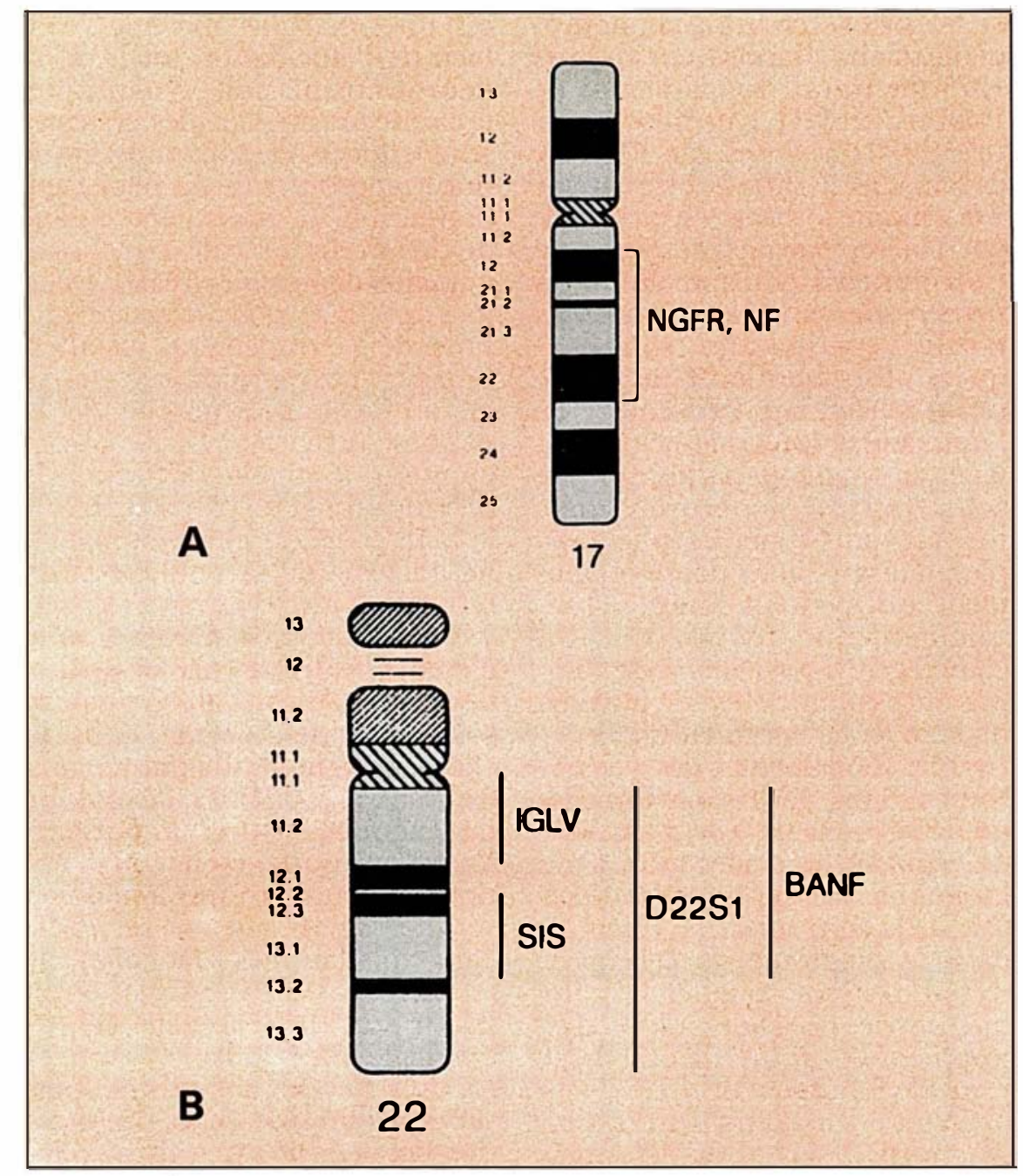

Figure 8. (A). Localisation chromosomique du gène NF (neurofibromatose) à proximité du gène NGFR sur lo chromosome 17. (B). Localisation chromosomique du gène BANF sur lo chromosome 22 (d'après [18]. NGFR $=$ nerve growth factor receptor; IGLV = immunoglobulin-chain variable reaction genes ; SIS = platelet-derived growth factor; $D 22 S 1$ = sonde moléculaire anonyme ; BANF = bilateral acoustic neurofibromatosis.

En revanche des notions communes sont établies : la forme et la situation des structures dérivées de la crête dépendent non seulement de la localisation d'arrêt des cellules en migration mais aussi de l'horaire et de la présence ou de l'absence de molécules spécifiques de la matrice d'origine extracellulaire. Les anomalies de développement peuvent résulter de la sorte aussi bien d'altérations des cellules de la crête que d'altérations de la matrice environnante elle-même.

Plus d'un siècle s'est écoulé depuis le mémoire de von Rec$\mathrm{m} / \mathrm{s} n^{\circ} 3$ vol. 4 , mars 88 classique dite VRNF (von Recklinghausen neurofibromatosis) est localisé par Seizinger et al. [17] sur le chromosome 17 en q12 $\rightarrow$ q22 à proximité du locus récepteur du NGF ; le gène localisé au sein des cellules cultivées à partir de neurinome de l'acoustique bilatéral (BANF), avec peu ou pas de taches café au lait, serait situé sur le bras long du chromosome 22 entre qll.l et 13.2 [18] (figure 8).

L'étude la plus passionnante est de toute évidence celle de Hinrichs et al., laquelle si elle devait être confirmée offre un modèle expérimental de la NF par la création d'une souche de souris transgénique (figure 9, page 165): le virus HTLV-I est connu pour provoquer la «paraparésie spastique tropicale $\%$. Le gène tat de HTLV-I induit des tumeurs qui évoquent fortement celles de la NF. Chez une souris transgénique de trois mois des tumeurs développées à partir des enveloppes nerveuses sont apparues et le phénotype est passe avec succès à travers trois générations [19].

S'agissant de neurocristopathies, il était attendu que leur potentiel oncogénique fréquent attirât l'attention des cancérologues, particulièrement ceux qui étudient les syndromes MEN (multiple endocrine neoplasia). Ces syndromes, transmis habituellement en dominance autosomique, et dont la source réside dans certaines cellules issues de la crête neurale, font partie intégrante de la neurogénétique [20]. Autre neurocristopathie non rare, la sclérose tubéreuse de Bourneville a donné lieu à moins de publications récentes. On connaît toutefois sa localisation génique sur le chromosome 9 [21] et Hausser et al. pensent que l'étude en microscopie électronique combinée avec la DOPA-réaction* des mélanocytes des conducteurs asymptomatiques de la mutation pourrait être révélatrice de leur état [22].

S'agissant de la migration neuronale qui crée le cortex cérébelleux

* DOPA : dihydroxyphénylalanine. 


\section{REFEERENCES}

34. Readhead C, Popko B, Takahashi N, et al. Expression of myelin basic protein gene in transgenic shiverer mice : correction of the dysmyelinating phenotype. Cell 1987; 48: 703-12.

35. Dautigny A, Mattei MG, Morello, et al. The structural gene coding for myelin associated proteolipid is mutated in jimpy mice. Nature 1986 ; 321 : 827-9.

36. Vanier MT, Wenger DA, Comly ME, Rousson R, Brady RO, Pentchev PG. Niemann-Pick disease group $C$ : clinical variability and diagnosis based on defective cholesterol esterification. A collaborative study on 70 patients. Clin Genet 1988 (sous presse).

37. Tsuji S, Choudary PV, Martin BM, et al. A mutation in the human glucocerebrosidase gene in neuropathic Gaucher's disease. New Engl J Med 1987; 316 : 570-5.

38. Sorge J, Kuhl W, West C, Beutler E. Complete correction of the enzymatic defect of type I Gaucher disease fibroblasts by retroviral mediated gene transfer. Proc Natl Acad Sci USA 1987 ; 84 : 906-9.

39. Gilliam TC, et al. A DNA segment encoding two genes very tightly linked to Huntington's disease. Science $1987 ; 238$ : 950-2.

40. Weyler NS, et al. Homozygotes for Huntington's disease. Nature 1987; 326: 194-7.

41. St-Georges-Hyslop Ph, Tanzi RE, Polinsky RJ, et al. Absence of duplication of chromosome 21 genes in familial and sporadic Alzheimer's disease. Science $1987 ; 238$ : 664-6.

42. Podlisny MB, Lee G, Selkoe DJ. Gene dosage of the amyloĩd $\beta$ precursor protein in Alzheimer's disease. Science 1987; 298: 669-71.

43. Mori H, Kondo J, Ihara Y. Ubiquitin is a component of paired helical filaments in Alzheimer's disease. Science 1987; 235: 1641-4.

44. Zubenko GS, Wusylko M, Cohen BM, Boller F, Teply I. Family study of platelet membrane fluidity in Alzheimer's disease. Science 1987 ; 238 : $539-42$

45. Marx JL. Alzheimer's drug trial put on hold. Science 1987 ; 238 : 1041-2.

46. Martin JB. Molecular genetics : applications to the clinical neurosciences. Science 1987 ; 238 : 765-72

47. Feil A. L'absence et la diminution des vertèbres cervicales. Êtude clinique et pathologique. Le syndrome de réduction numérique cervicale. Thèse médecine, Paris, 1919.

48. Ogden JA, Lee KE, Conlogue JG, Barnett JS. Prenatal and postnatal developmen of the cervical portion of the spine in the short-finned pilot whale Globicephala macrorinynha macrorhynea. Anat Rec 1981; 200 : et le cortex cérébral, les neurogénéticiens disposaient depuis $1975 \mathrm{du}$ travail fondamental de Pasco Rakic [23]. Une mise à jour tirée de 90 références, que l'édíteur de la revue qualifie à juste titre de «fascinante», nous est apportée par Harvey Sarnat [24]. Son titre trop limitatif pourrait donner à penser que seules les migrations tardives, périnatales, sont évoquées alors que l'auteur fait le point actuel sur l'ensemble de l'ontogenèse cérébrale et cérébelleuse normale et pathologique, sous contrôle génétique, mais insistant aussi sur les phénocopies, d'origine infectieuse notamment, qui peuvent simuler cliniquement une génopathie. En conseil génétique l'affaire est d'importance comme elle l'est aussi en matière de lissencéphalie [25].

Le rôle fondamental des péroxysomes dans la formation des guides gliaux, lié à leur capacité de dégrader les acides gras à très longue chaîne, explique les désordres de la corticalisation cérébrale dans le syndrome de Zellweger, récessif autosomique, et la polymicrogyrie de l'adrénoleucodystrophie liée à l'X. L'excellente revue de Schutgens et al. [26] vient d'autant mieux à son heure qu'un diagnostic prénatal est désormais possible dans ces deux mutations. - Synaptogenèse. Si l'étude des mutants de la souris, type Staggerer notamment, ne semble pas avoir encore révélé tous les secrets de la synaptogenèse cérébelleuse, ce qui nous aideraient à comprendre et à traiter les agénésies ou les hérédodégénérescences cérébelleuses, le travail très original de C. Sotelo et R.M. Alvarado-Mallart [27] mérite une lecture attentive. Ces deux chercheurs de l'Inserm semblent avoir réussi à rétablir chez le mutant Purkinje cell degenerations (Pcd) une «circuiterie» cérébelleuse fonctionnelle par des greffes de cellules cérébelleuses embryonnaires. D'autres chercheurs, en France, ont axé leurs études sur l'amyotrophie spinale infantile dont il est possible qu'elle relève d'un défaut de la synaptogenèse. Dans le tout autre domaine de certaines maladies mentales, c'est bien de l'élucidation totale de la neurotransmission synaptique, notamment au sein des systèmes adrénergique et dopaminergique que viendront clés du dépistage, prévention et traitement curatif de la maladie (ou psychose) maniaco-dépressive (PMD). Deux loci candidats pour le gène PMD sont déjà solidement installés, l'un sur le chromosome X, en q27 lié au gène polymorphe de la synthèse du facteur IX de la coagulation, à la suite du travail de Mendlewicz et al. [28], l'autre sur le bras court du chromosome 11 , lié au gène de la synthèse de la tyrosine-hydroxylase (TH) par J.A. Egeland et Gehrard à la suite d'études réalisées chez des sujets de souche Amish [29]. On sait le rôle de cette enzyme dans la chaîne catécholaminergique $(\mathrm{m} / \mathrm{s}$ $n^{\circ} 5$, vol. 3, p. 301). Le laboratoire de Jacques Mallet à Gif-surYvette, travaillant en collaboration avec un groupe londonien sur des sujets atteints de PMD dans trois familles islandaises, ne retrouve pas cette association et insiste sur l'hétérogénéité génétique de la PMD [30].

La diversité des mécanismes possibles à l'origine de la prolifération des dendrites (les phénylcétonuriques, les hypothyroïdiens congenitaux souffrent d'une particulière incapacité en ce domaine), de la création des synapses, de leur «stabilisation sélective » (J.-P. Changeux), de leur aptitude à émettre, à recapturer, a stocker* les molécules des neurotransmetteurs offre aux neurobiologistes un champ d'investigations infini [31].

\section{Myélinisation}

Cette phase du développement du système nerveux central et périphérique des vertébrés a l'avantage de disposer, pour son étude, de l'existence de modèles animaux de l'espèce murine [32].

\footnotetext{
* En matière de stockage, le róle dévolu d̀ la synapsine $1\left(\mathrm{~m} / \mathrm{s} \mathrm{n}^{\circ} 7\right.$, vol. 3, p. 422) appelle l'attention sur cette protéine du cytosquelette.
} 

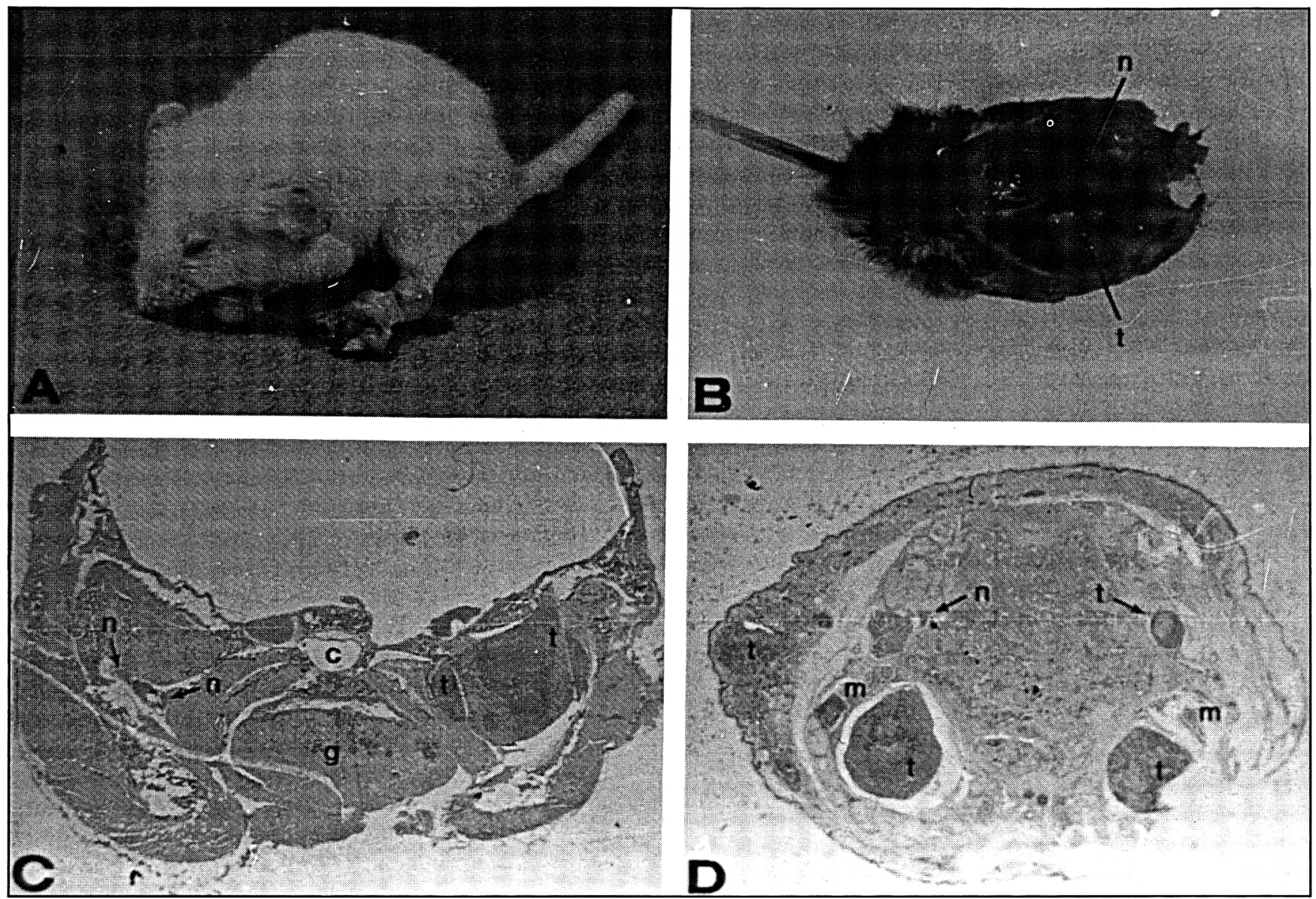

Figure 9. Lo souris neurofibromateuse de Hinrinchs [19]. (A). Neurofibromes du front, des pattes, des oreilles et de la queue. (B). Base du crâne. Tumeur du nerf trijumeau. (C). Section coronale de la tête (c = cavité nasale; $g=$ langue ; $n=$ nerfs ; $t=$ tumeur nerveuse). (D). Coupe de la queue $(n=$ nerf; $m=$ muscle ; $t=$ tumeur nerveuse).

La souris mutante, shiverer, homozygote récessive pour le gène muté shi est épileptique et meurt précocément. La protéine basique de la myéline (MBP) fait défaut. Chez la souris, un seul gène situé sur le chromosome 18 code en réalité, à la suite d'un épissage alternatif au niveau d'un de ses sept exons, pour plusieurs types de molécules allant de 14 à $21 \mathrm{kDa}$ (kilodalton). Roach et al. [33] ont démontré que le phénotype shiverer était provoqué par une délétion du gène qui fait disparaître le gène entier, à l'exception de l'exon I et du début de l'intron. Readhead et al. [34] par microinjection dans l'œuf fertilisé du gène MBP sauvage ont «guéri » des souris mutantes qui ne tremblent plus et ont une durée de vie $\mathrm{m} / \mathrm{s} n^{\circ} 3$ vol. 4, mars 88 normale $\left(m / s n^{\circ} 6\right.$, vol. 3, p. 359). La souris mutante jimpy présente une affection génétique de la myélinisation dont la sémiologie, l'histopathologie et la transmission héréditaire (liée à l'X) n'est pas sans évoquer la maladie humaine décrite par PelizaeusMerzbacher (McKusick $\left.n^{\circ} 31160\right)^{*}$. Le locus jimpy est probablement le même que celui de la lipophiline (ou protéolipide protéine, PLP), constituant principal de la myéline centrale synthétisé par les oligodendrocytes. Des chercheurs français [35] ont montré l'effondrement de son ARN chez les mutants $\left(\mathrm{m} / \mathrm{s} n^{\circ} 9\right.$, vol. 2, p. 526).

* In McKusick V. Mendelian-inhevitance in man, 7e ed. The John Hopkins University Press, 1986, 1741 pages.
Les recherches progressent aussi dans le cadre des erreurs innées du métabolisme (TableauII, page 166) notamment dans le domaine du diagnostic prénatal. A Lyon, M.-T. Vanier et al. poursuivent leur dissection des origines de la maladie de NiemannPick [36]. La maladie de Gaucher connaît trois formes cliniques, le type 1 sans composante neurologique au contraire des types 2 et 3. Le séquençage par $\mathrm{S}$. Tsuji et al. [37] d'un gène clôné provenant d'un malade de type 2 a montré l'existence d'une mutation ponctuelle leu-pro à l'origine du déficit en glucocérébrosidase. L'équipe californienne d'Ernest Beutler, après avoir souligné les échecs de la substitution enzymatique, de la greffe splénique ou 
Tableau II

EXEMPLES D'ERREURS INNÉES DU MÉTABOLISME (A VERSANT NEUROLOGIQUE)

DONT LE DÉFAUT GÉNÉTIQUE A ÉTÉ LOCALISÉ ET/OU EXPLIQUÉ (D’APRĖS [46])

\begin{tabular}{|c|c|c|}
\hline Affection & Chromosome & Défaut génétique \\
\hline \multicolumn{3}{|l|}{ Dominant autosomique } \\
\hline Polyneuropathie amyloïdosique familiale & $18 \mathrm{q} 11.2 \rightarrow 12.1$ & $\begin{array}{l}\text { Substitution d'une paire de bases sur l'ARNm } \\
\text { de la transthyrénine }\end{array}$ \\
\hline \multicolumn{3}{|l|}{ Récessif autosomique } \\
\hline Maladie de Gaucher & 1 q 21 & $\begin{array}{l}\text { Substitution d'un amino-acide dans la gluco- } \\
\text { cérébrosidase }\end{array}$ \\
\hline Gangliosidose à GM1 & 3 p 1 & Partiellement caractérisé \\
\hline $\begin{array}{l}\text { Gangliosidose à GM2 } \\
\text { - Tay-Sachs (type 1) } \\
\text { - Sandhoff (type 2) }\end{array}$ & $\begin{array}{c}15 \text { q } 22-q 25 \\
5 \text { q } 13\end{array}$ & $\begin{array}{l}\text { Mutation d'un gène codant pour la chaîne } \alpha \\
\text { de l'hexosaminidase } \\
\text { Mutation d'un gène codant pour la chaîne } \beta \\
\text { de l'hexosaminidase }\end{array}$ \\
\hline Maladie de Wilson & 13 q 14.11 & Inconnu \\
\hline \multicolumn{3}{|l|}{ Lié à l'X } \\
\hline Adréno-leucodystrophie & $X q 27-q 28$ & $\begin{array}{l}\text { Métabolisme des acides gras à longues } \\
\text { chaînes }\end{array}$ \\
\hline Lesch-Nyhan & Xq 27 & Déficience en HPRT \\
\hline Pelizaeus-Merzbacher & Xq 21 - q 22 & $\begin{array}{l}\text { Déficit en protéine protéolipidique de la } \\
\text { myéline }\end{array}$ \\
\hline
\end{tabular}

GM1 = monosialoganglioside 1; GM2 = monosialoganglioside 2; HPRT = hypoxanthine-guanine phosphoribosyltransférase .

rénale, et la fragilité des résultats de la greffe de la moelle osseuse dans la maladie de type 1 révèle qu'il a été possible de corriger complètement in vitro le défaut enzymatique en utilisant un vecteur rétro-viral qui restitue la fonction normale chez le fibroblaste en culture du patient [38]. La question de la prédisposition génétique à la sclérose en plaques a été longuement débattue depuis plus de dix années. Un article de synthèse est paru récemment dans médecine/sciences (Génétique de la sclérose en plaques, $m / s n^{\circ} 1$, vol.3, p.13). Joèl Oger, Olivier Sabouraud et Bary Arnason ont eu le grand mérite d'éclairer ce chapitre complexe et de décrire les recherches les plus récentes à propos de la plus fréquente des maladies neurologiques. Le lecteur est invité à s'y reporter.

\section{Maintenance \\ et pré-sénescence}

Maladie de Huntington (HD). Quatre ans après, presque jour pour jour, l'annonce par Gusella et al.del'isolement de la sonde G8, les mêmes chercheurs auxquels se sont joints des chercheurs londoniens et de l'EMBO (Heidelberg) annoncent qu'ils détiennent une «candidate pour le site» : il s'agit de la sonde anonyme D4S43: 8 RFLP* $^{*}$ sont identifiés dans trois vastes familles, ainsi que deux seg- ments codants indépendants l'un de l'autre [39]. La région est située entre le télomère et G8 rebaptisé D4S10. Un tel ciblage va conforter la démarche prédictive (porteurs encore asymptomatiques et diagnostic prénatal) mettant les conseillers médicaux au pied du mur.

Nancy Wexler et al. [40] poursuivant l'étude généalogique, clinique et moléculaire des lignées lymphoblastoïdes de la gigantesque famille du lac Maracaïbo (Vénézuela) ont soigneusement isolé des sujets à forte probabilité

* RFLP: restriction fragment length poly. morphism (m/s suppl. $\mathrm{n}^{\circ} 7$, vol. 3, p. 4). 
d'être porteurs du gène muté en double-dose. L'expression clinique ne diffère en aucune manière de celle qui est habituelle chez les hétérozygotes. L'intérêt de ces cultures cellulaires dépourvues de tout allèle normal est à souligner, dans l'optique d'une recherche du défaut moléculaire à la source de l'affection.

En matière de tests prédictifs, l'heure paraît être à la réflexion et à la prudence. La section européenne de l'IHA (international Huntington's association), l'Association Huntington de France** diffusent auprès des malades et de leurs familles les informations et les conseils nécessaires.

Maladie d'Alzheimer. Dans le numéro de mai 1987 de médecine/sciences $\left(\mathrm{m} / \mathrm{s} n^{\circ} 6\right.$, vol. 3 , p. 256), P.-M. Sinet et al. ont eu recours au procédé d'un addendum pour incorporer dans la synthèse qu'ils publiaient sur «Trisomie 21 et maladie d'Alzheimer » six références d'articles parus depuis la remise de leur manuscrit à l'éditeur. L'inflation se poursuit et c'est une sorte de bataille ouverte à laquelle on assiste, notamment à la suite de la sortie du numéro de Science du 30 octobre 1987. Les groupes de Gusella et de Tanzi [41], de Podlisny [42] par des abords différents (études de RFLP ou du dosage génique) pensent apporter la preuve que l'hypothèse selon laquelle le défaut génétique qui crée la maladie est la duplication d'un segment du chromosome 21 n'est pas vérifiée.

Simultanément ou presque deux autres voies de recherches s'ouvrent: l'analyse immunologique plus fine des paires de filaments en hélice (PHF) met en évidence selon Mori et al. [43] la présence d'épitopes d'ubiquitine, élément hautement conservé nécessaire au fonctionnement du système protéolytique ATP-dépendant. Pour être dégradées certaines protéines ont besoin d'être «ubiquitinisées » et c'est un défaut de cette

\footnotetext{
* Association Huntington de France, 119, rue Manin, 75019 Paris, France.

$\mathrm{m} / \mathrm{s} n^{\circ} 3$ vol. 4, mars 88
}

molécule qui entraînerait dans la maladie d'Alzheimer la formation des dépôts amyloïdes, tout au moins selon ces auteurs. Enfin, Zubenko et al. pensent avoir mis au point [44], par l'étude en fluorescence d'un dérivé de l'hexatriène sur des membranes plaquettaires marquées, un test prédictif de la maladie chez les apparentés asymptomatiques des sujets atteints.

Si ce dernier travail devait être confirmé, les conseillers génétiques se trouveraient confrontés aux mêmes situations qu'ils rencontrent lorsqu'ils sont consultés par les familles de sujets atteints de maladie de Huntington : fautil ou non dire à un homme (ou à une femme) de 40, 50 ans qu'il est menacé par une démence dont on ignore à ce jour et la cause précise et donc bien entendu le traitement préventif et curatif [45]?

\section{Conclusion}

Cette synthèse, qui a suivi le fil directeur de la chronologie du développement du système nerveux central, a tenté d'éviter le piège d'être assimilée à un catalogue. Celui-ci eût, de toute manière, été incomplet puisque, de parti pris, il n'a pas été question des maladies musculaires d'origine génétique (que les neurologues cliniciens considèrent depuis le XIX' siècle comme l'une de leurs chasses réservées) ni des maladies du système nerveux périphérique (alors que, par exemple, le démembrement des polynévrites dites «familiales » est en cours, à la suite de découvertes récentes de grand intérêt).

Enfin et surtout, la génétique du fonctionnement synaptique lors des troubles de l'équilibre thymique n'a été qu'évoquée [28-31]. Les troubles du comportement ne l'ont pas été, puisque la molécule de la folie n'existe pas. Mais les neuro-généticiens infiltrent désormais largement un domaine jusqu'alors réservé aux psychiatres. On ne peut que s'en féliciter, pour les uns et pour les autres

\section{Summary}

The mechanisms underlying CNS malformations has become enlightened by knowledge of normal CNS development. Thus, notochord (diastematomyelia, Klippel-Feil syndrome) and neural tube defects (anencephalia, spina bifida) are the consequence of perturbation of neuro-ontogenesis between the third and the fourth week of fetal life. Interruption of neuronal multiplication (microcephalia vera, corpus callosum agenesis, holoprosencephalia) and neuronal migration to neural crest (NF, TS) or cortical plate (lissencephalia) results likely from disregulated mechanisms of cell to cell interaction and inherited genetic information in cellular differentiation. Recent advances in metabolic disorder and molecular biology showed up the role of peroxisomes (Zellweger syndrome), oncogenes (int-1, c-fos) and growth factors (NGF) in developing CNS. The mechanisms that regulate many important changes at brain synapses has led to the recognition of NMDA receptors in synaptic plasticity in the adult (learning, memory) and central neural development. Identification of particular human diseases (Huntington, Alzheimer) and animal mutant models are valuable initial steps towards elucidating the molecular basis of neuronal maintenance, aging and myelination.

\section{TIRÉS A PART}

J.-M. Robert: Hôtel-Dieu, service de génétique, place de l'Hôpital, 69002 Lyon, France. 